\title{
Cryogenic VPH gratings for the CELT/TMT
}

Sebastien Blais-Ouellette, Dani Guzman, Amal Elgamil, Richard Rallison

Sebastien Blais-Ouellette, Dani Guzman, Amal Elgamil, Richard Rallison, "Cryogenic VPH gratings for the CELT/TMT," Proc. SPIE 5494, Optical Fabrication, Metrology, and Material Advancements for Telescopes, (24 September 2004); doi: 10.1117/12.552185

Event: SPIE Astronomical Telescopes + Instrumentation, 2004, Glasgow, United Kingdom 


\title{
Cryogenic VPH gratings for CELT/TMT
}

\author{
Sébastien Blais-Ouellette ${ }^{a, b}$, Dani Guzman ${ }^{a}$, Amal Elgamil $^{a}$, Richard Rallison $^{c}$, \\ ${ }^{a}$ California Institute of Technology, 1200 E. California blvd., Pasadena, CA, USA \\ ${ }^{b}$ Photon etc. inc., 432 boul. des Prairies, Laval, Canada \\ ${ }^{c}$ Wasatch Photonics, 1305 North 1000 West \#120 Logan, Utah 84321, USA
}

\begin{abstract}
Characterization of Volume Phase Holographic gratings at cryogenic temperatures have been conducted using a new test facility at Caltech. The new test bench includes a cryostat that allows large angles for incident and diffracted light. Gratings under tests are shielded from thermal background, and precisely and uniformly temperature controlled. Preliminary results are presented and show little temperature dependence of the efficiency function.
\end{abstract}

Keywords: astronomy, instruments, near infrared, visible, cryogenic, VPH, grating

\section{INTRODUCTION}

Volume-Phase Holographic (VPH) gratings represent a very efficient alternative to classical surface-relief gratings. Many of these devices are currently in use in various visible wavelength spectrographs on telescopes such as VLT, AAT and Gemini. They are usually made of di-chromated gelatin sandwiched between two glass plates. Index of refraction is modulated across the gelatin as shown in Figure 1.

One of the most interesting features of Volume Phase Holographic (VPH) gratings is the possibility of adjusting the efficiency curve, or "blaze" function, by varying the angle of incidence. Indeed, while their dispersive properties are identical to that of classical gratings, their diffracted energy distribution is governed by Bragg's law, as for X-rays in a crystalline structure. In the case where the modulation fringes are perpendicular to the grating surface (Figure 1a), the Bragg condition follows,

$$
m \nu \lambda=2 n_{\text {grating }} \sin \alpha_{\text {grating }}
$$

and from Snell's law

$$
m \nu \lambda=2 \sin \alpha_{\text {air }}
$$

where $\mathrm{m}$ is the order of diffraction, $\nu$ is the fringe frequency in the grating, $\lambda$ is the wavelength, $\mathrm{n}$ is the average index of refraction, and $\alpha$ is the angle of incidence. Radiation that departs significantly from the Bragg condition passes through the grating undiffracted. This tunability can be advantageously used in spectrographs ${ }^{1}$ using an articulated camera for example.

As an example of their usefulness figure 2 presents a comparison of optical design using traditional grism and an equivalent VPH and prisms assembly. Such an assembly contributes to reduce the costs and risks of building a transmissive multi-object spectrograph for a giant telescope, Keck in this case. Indeed, the development of large sapphire prisms is an important cost and risk driver for a conventional solution.

VPH gratings have very high efficiency and can be optimized for any wavelength. According to Kogelnik's coupled wave analysis, ${ }^{2}$ efficiency of thick gratings with unslanted fringes is approximately, for the two planes of polarization:

$$
\begin{gathered}
\eta_{s}=\sin ^{2}\left(\frac{\pi \Delta n}{\lambda} \frac{d}{\cos \alpha}\right) \\
\eta_{p}=\sin ^{2}\left(\frac{\pi \Delta n \cos (2 \alpha)}{\lambda} \frac{d}{\cos \alpha}\right)
\end{gathered}
$$

Send correspondence to sbo@caltech.edu 


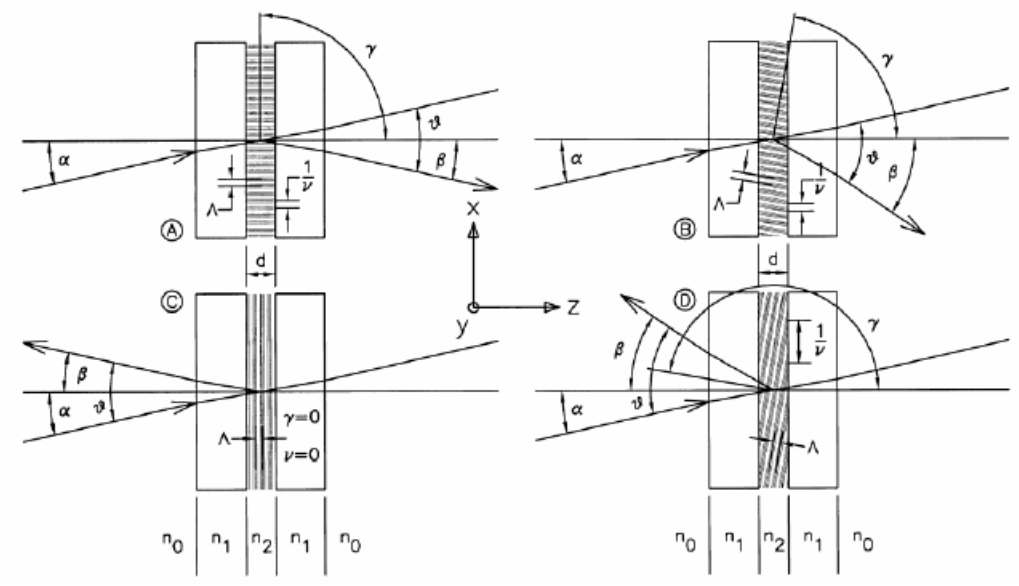

Figure 1. Four possible fringe structures for VPH gratings : (A) Littrow transmission grating. (B) Non-Littrow transmission grating. (C) Non-dispersive reflection grating (notch filter). (D) Dispersive reflection grating.
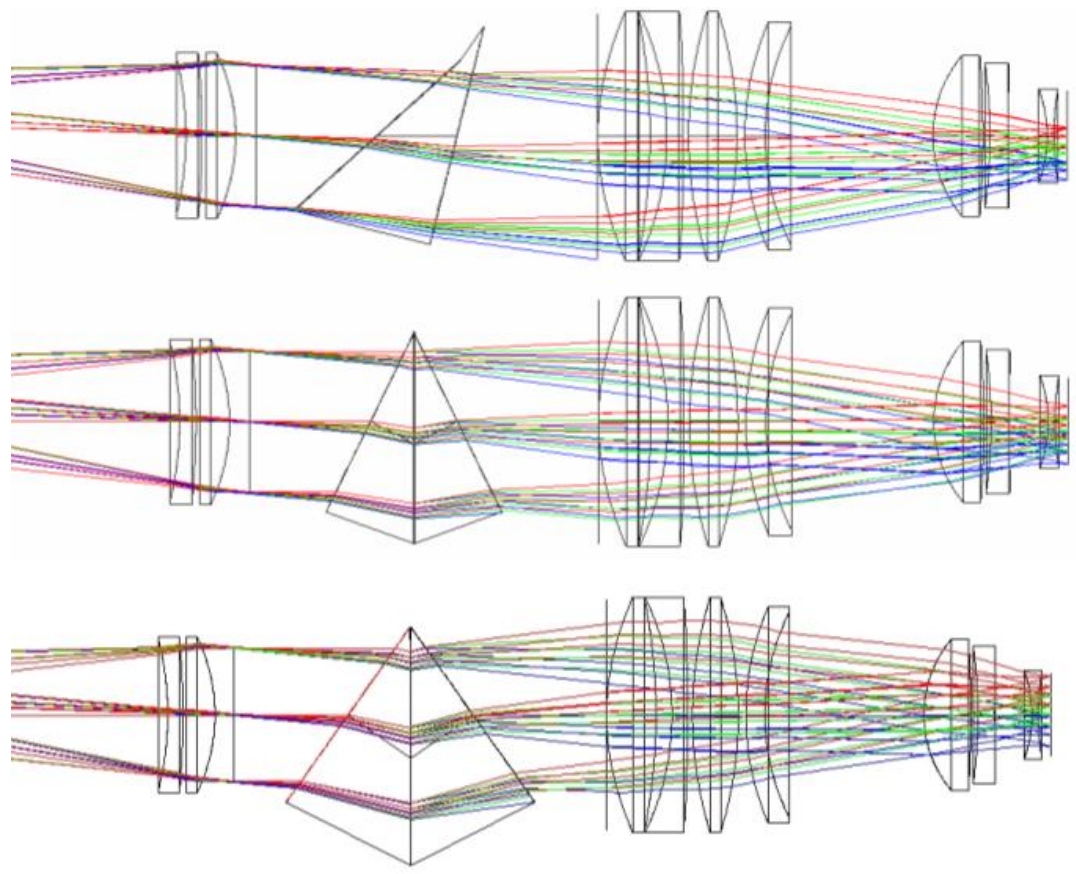

Figure 2. Top - Grating and sapphire prism. Middle - Equivalent VPH and fused silica prisms. Bottom - Higher dispersion VPH and fused silica prisms.

where $\alpha$ is the angle of incidence in the air, $\nu$ is the fringe frequency, and $\mathrm{d}$ the depth of the grating structure. Also, the wavelength bandwidth is given by:

$$
\frac{\Delta \lambda_{F W H M}}{\lambda} \simeq \frac{\cot \alpha}{\nu d}
$$

As a practical example figure 3 shows the efficiency function of three VPH gratings optimized for the $J, H$ and $K$ band. 


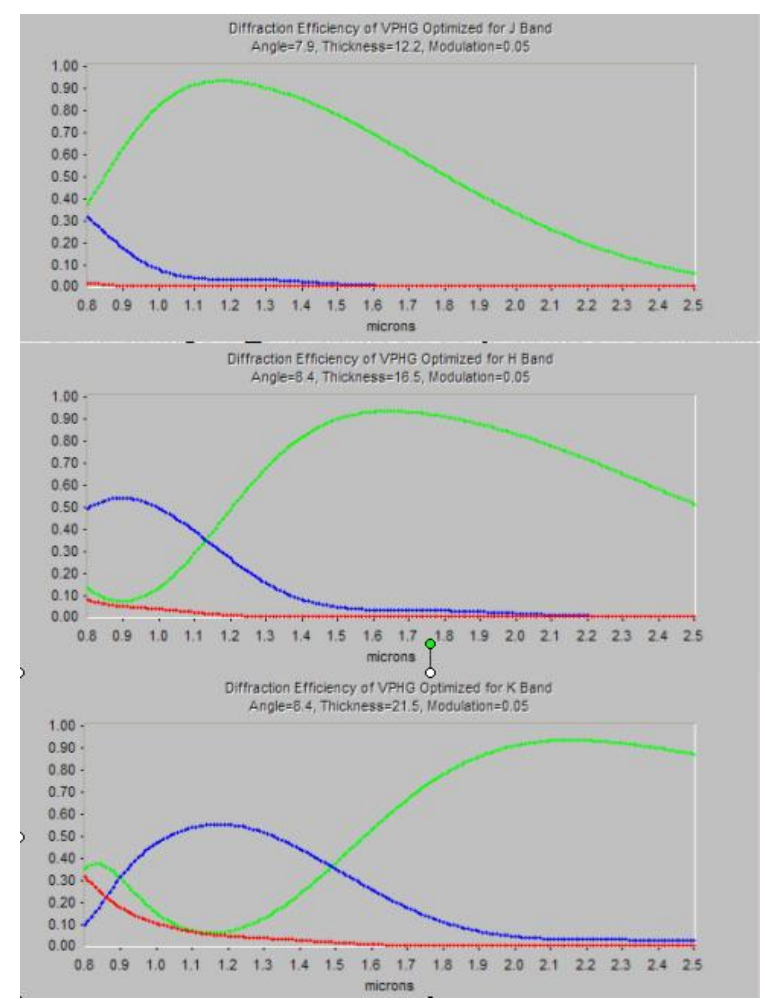

Figure 3. Efficiency curve for three VPH gratings optimized for $J$ (top), $H$ (middle), and $K$ (bottom).

\section{CRYOGENIC TEST BED}

Considering the importance of near-infrared spectroscopy and its sensitivity to thermal radiation, it is of great interest to understand the behavior of these gratings at cryogenic temperature. To do so, we have built a test facility that includes a cryogenic chamber and fully automated moving stages and detector for the IR and visible light. The facility is also used to test CCDs and IR arrays detectors as described in Guzman 2004. ${ }^{3}$ Figure 4 describes the test bench in the mode allowing efficiency testing of cold optical components. It is composed of a monochromatic light source collimated on the optical component, the VPH in this case, a cryostat mounted on a rotation table, and two detectors mounted on a semi-circular rail. The monochromator (including an order sorter filter wheel and a neutral density filter wheel) the rotation table, and the detector rail are motorized and controlled via a Labview application. In addition, temperature inside the cryostat is monitored by five sensors and controlled by two resistors through the same Labview application. A polarizer is also placed at the exit port of the monochromator.

The cryostat head (figure 5) has large windows to allow incident and diffracted light to form angles up to $90^{\circ}$. One of the consequence of that design is the large amount of outside thermal radiation hitting the optical element inside the cryostat. This causes not only a rise in temperature but also a temperature gradient from the center to the cooled edge of the optical element. To alleviate this cooling problem, a pair of cooled windows shields the optical sample from most of outside thermal radiation. These fused silica windows act as a filter, reflecting radiation above $2.5 \mu \mathrm{m}$. The optical element is then only subject to the blackbody radiation of these cooled windows and to the outside radiation up to the windows cut-off wavelength.

\section{METHODOLOGY}

About 20 samples were provided by Wasatch Photonics. Three sets of respectively 494, 685, and 1095 lines $/ \mathrm{mm}$ are divided in samples with optimal index modulation for diffraction efficiencies above $90 \%$, and samples over 


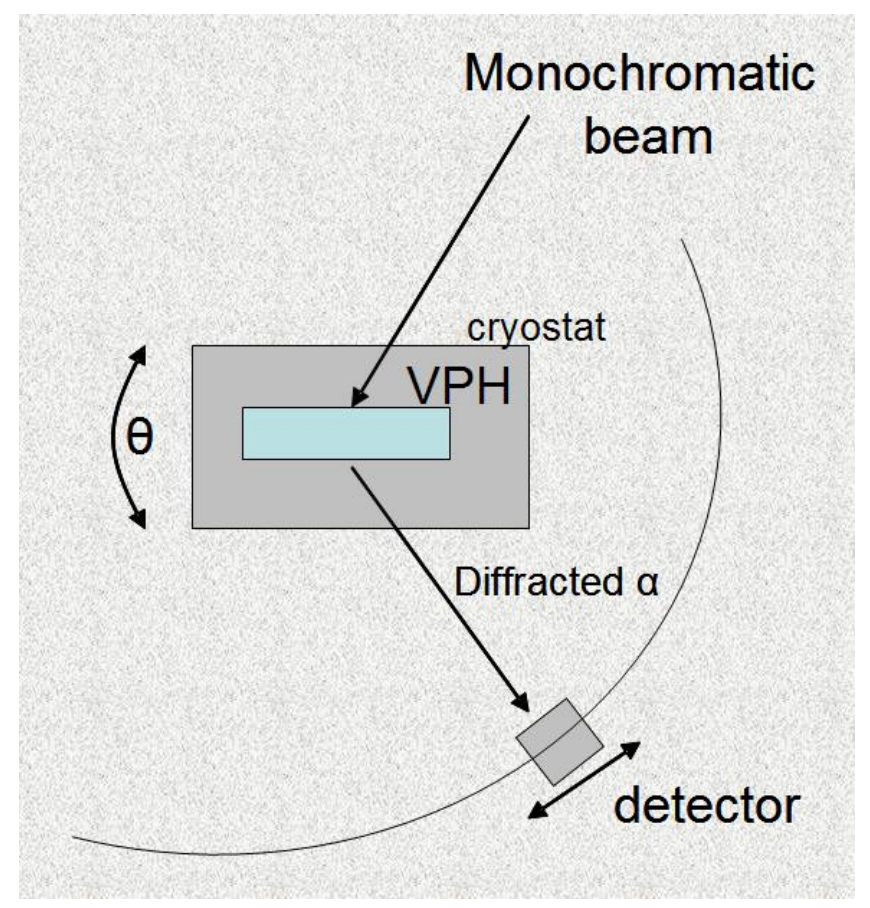

Figure 4. Schematic view of the test bed. A monochromatic beam is diffracted by the grating and measured by the detector. Both the grating and the detector can move to change the angles of incidence and diffraction.

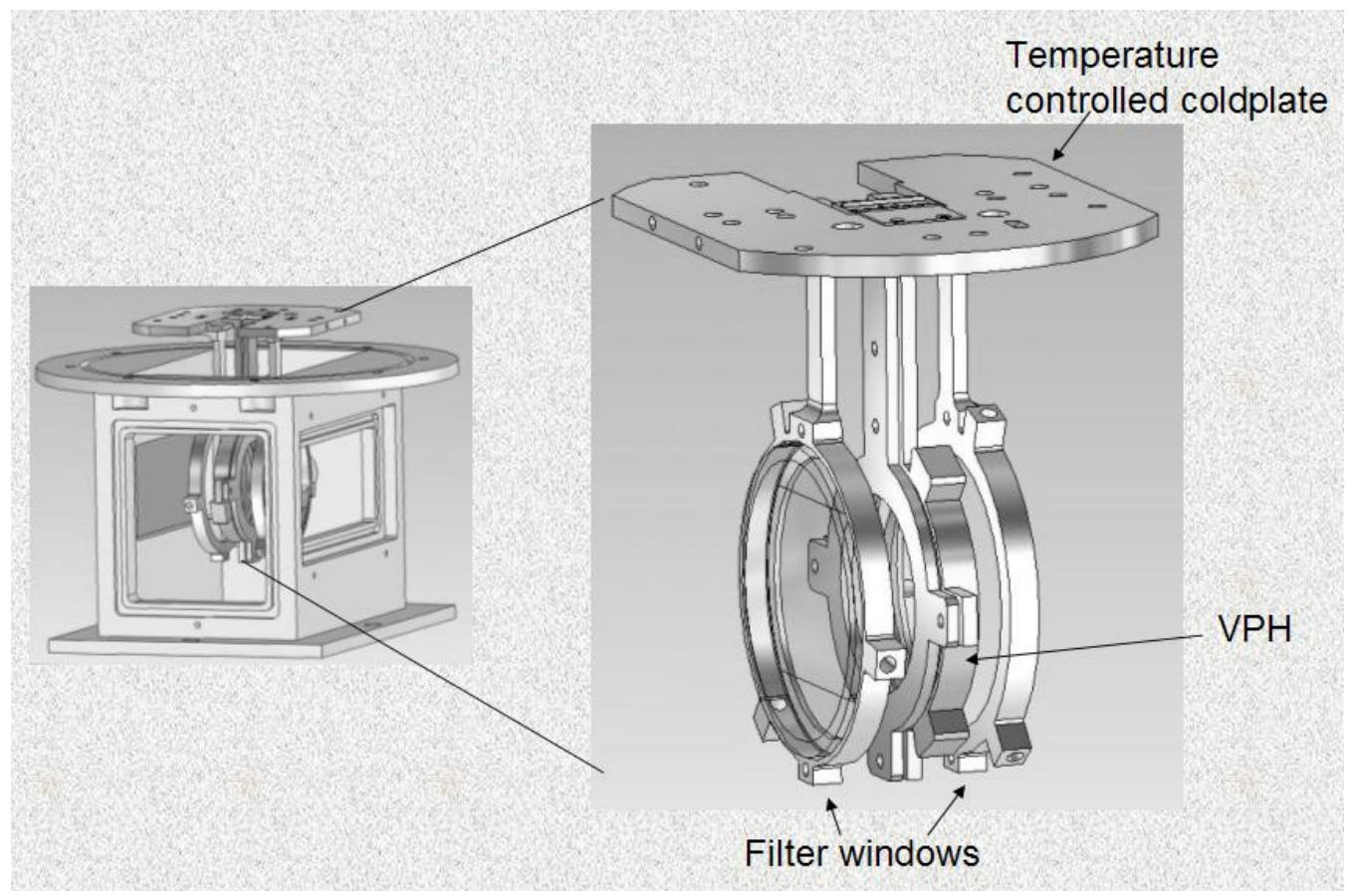

Figure 5. Cryostat head containing the optical element under test, the VPH in this case. Outer windows allow up $90^{\circ}$ between incident and refracted beam. The sample is sandwiched between two thermal shield windows. Temperature is monitored at multiple point in the cryostat and controlled by two resistors. 


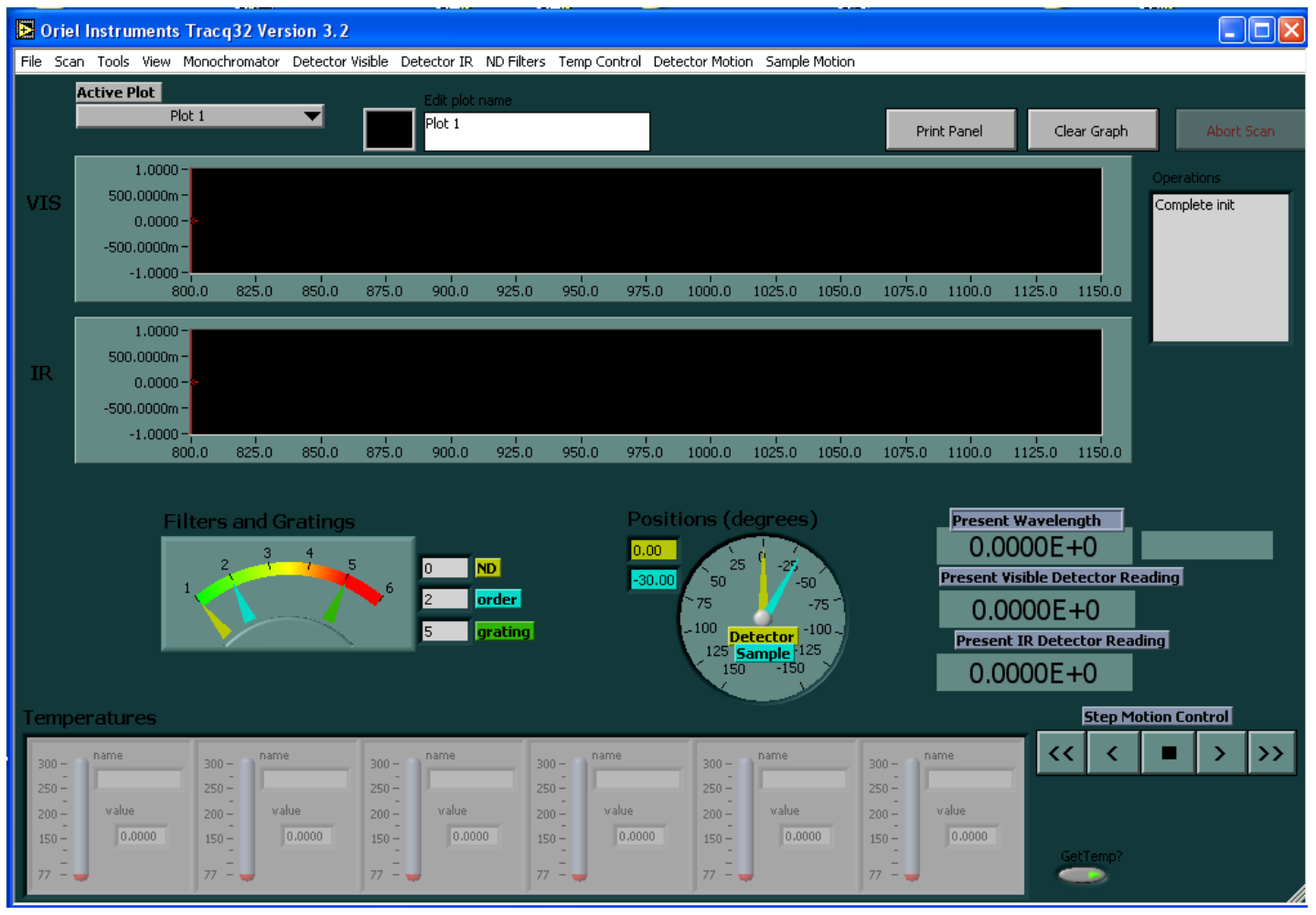

Figure 6. Main interface of the Labview application that controls the test bed.

or under-modulated. The goal of this distribution is to be able to study, among other things, the effect of temperature on index modulation.

To ensure a complete understanding of the test results, an alignment procedure was devised to account for any misalignment between the VPH and the cryostat windows. In addition, the testing procedure scans through all angles of incidence and diffraction to track any residual misalignment or discrepancy in the results.

A Labview application (figure 6) controls the various scans and motions. Any region of the parameter space composed of wavelength, light intensity, sample angle, detector angle, and temperature can be scanned in an automated manner. This gives a important flexibility to reduce the testing time while obtaining complete and robust results.

For the first tests presented here a spectrum was taken at each angular position of the detector. The scan was repeated for many sample positions, effectively scanning incident and diffraction angles. The process was done at room temperature and at the coldest stable temperature, without heating. The temperature reached by the VPH was first characterized with a dummy sample where sensors were glued near the center and on its aluminum holder. After a settling time of about 2 hours the difference between the two sensors is about $17^{\circ} \mathrm{C}$. That represents a strong upper limit for the gradient in the sample considering that the thermal contact between the glass and the holder is far from perfect.

\section{PRELIMINARY RESULTS}

As a first sample we tested a 685 lines/mm, optimally modulated. According to Wasatch photonics, these gratings are optimized for $21.37^{\circ}$. Using equations 2 we expect the efficiency to peak around 1.064 micron at this angle. To cover the peak efficiency region in a limited time we used the parameter showed in table 1.

Figure 7 shows the results for three sample positions. Each small curve represents a spectrum taken at a given detector angle in S-polarization. The efficiency curve, equivalent to the classical grating "blaze" function, 
Table 1. Scan parameters for a $685 \mathrm{l} / \mathrm{mm}$ sample.

\begin{tabular}{|l|c|c|}
\hline \hline Temperature: & $300 \mathrm{~K}$ & $105 \mathrm{~K}$ \\
\hline Wavelength: & $800 \mathrm{~nm} \rightarrow 1150 \mathrm{~nm}$ & $800 \mathrm{~nm} \rightarrow 1150 \mathrm{~nm}$ \\
Detector Angle: & $34^{\circ} \rightarrow 52^{\circ}$ & $36^{\circ} \rightarrow 50^{\circ}$ \\
Sample Angle: & $18^{\circ} \rightarrow 25^{\circ}$ & $19^{\circ} \rightarrow 24^{\circ}$ \\
\hline
\end{tabular}

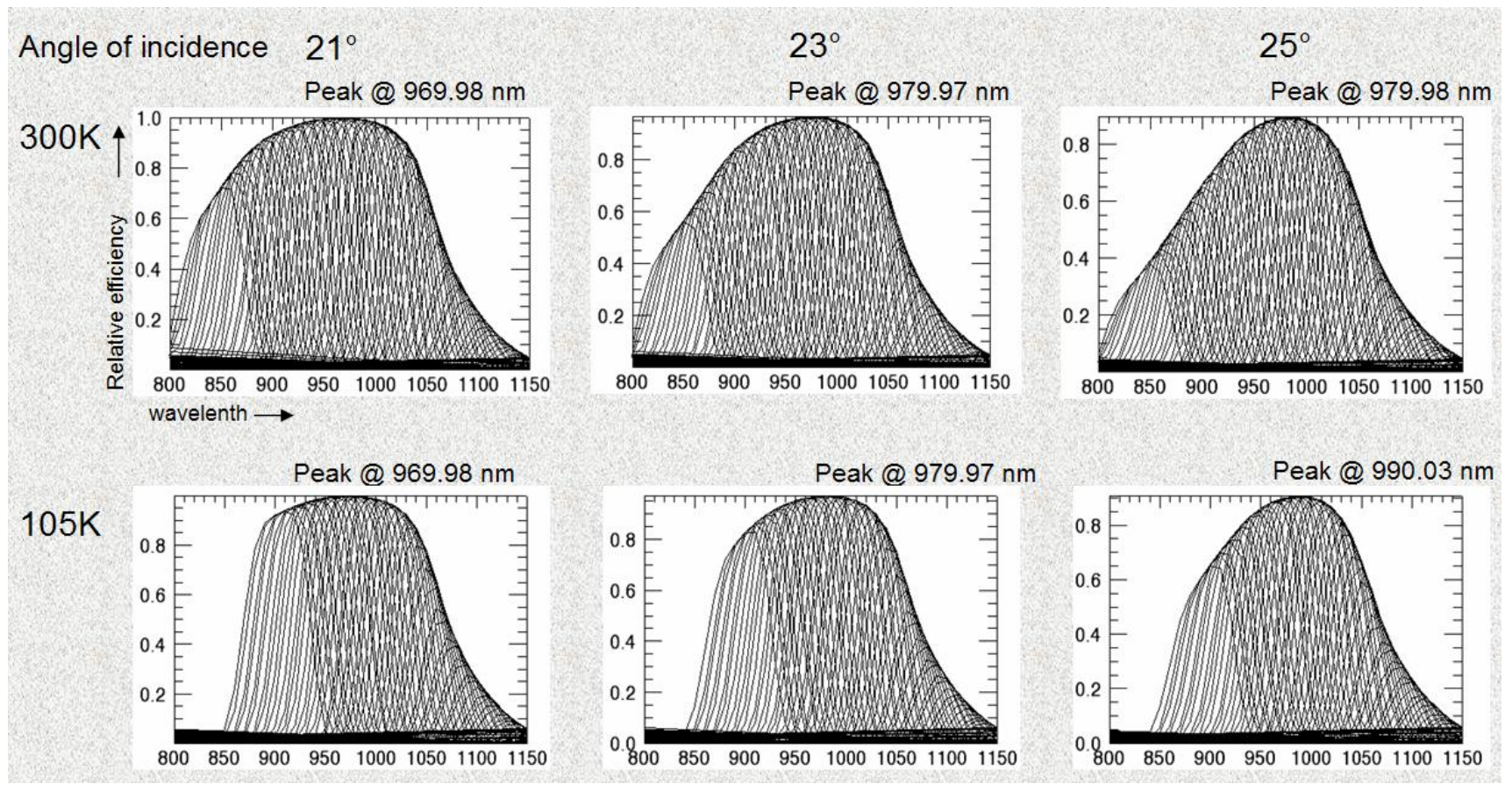

Figure 7. Results from efficiency tests.

is obtained by plotting the maximum of each spectrum, or their envelope. The preliminary results are shown as an indication of the global trends. More data and better calibration are needed to draw precise conclusions.

At $21^{\circ}$, close to the optimum angle, the efficiency peaks at a wavelength of $970 \mathrm{~nm}$, about $85 \mathrm{~nm}$ below the expected value. As expected, it shifts toward the red at higher angle of incidence. This is true for warm and cold tests but for the $25^{\circ}$ degree sample position where the blue part of curve drops but the red part stays the same, peaking at the same wavelength than at $23^{\circ}$.

The comparison of warm and cold tests are quite surprising. One would expect the fringes density to get higher as the glass cools and contracts. The reality is a quite different. The efficiency curves are very similar at $105 \mathrm{~K}$ and $300 \mathrm{~K}$. The peaks are at similar wavelengths. Only a slight narrowing of the curves can be observed. This behavior is unexpected but could be due to a higher than previously measured thermal gradient across the sample. More thermal calibrations are necessary to draw any definitive conclusion.

\section{CONCLUSION}

Preliminary results at $300 \mathrm{~K}$ and $105 \mathrm{~K}$ tend to show that the impact of temperature on the efficiency profile of VPH is surprising low. These results could be explained by a higher than expected temperature gradient across the sample although it is unlikely to suffice to explain such stability.

To have a clearer picture and to study all the possible problems, a larger part of the parameter space needs to be explored with higher precision. In addition to angles, wavelength, and temperature other variables like line 
frequency, modulation, variation across sample surface, and polarization will be explored to fully understand the cryogenic behavior of VPH gratings.

Once these small samples fully understood, it will be possible to build and optimize a larger gratings for a fully cryogenic infrared instrument.

\section{ACKNOWLEDGMENTS}

The work by EW and WvB was performed under the auspices of the U.S. Department of Energy by the University of California, Lawrence Livermore National Laboratory under contract No. W-7405-Eng-48.

\section{REFERENCES}

1. S. C. Barden, J. B. Williams, J. A. Arns, and W. S. Colburn, "Tunable Gratings: Imaging the Universe in 3-D with Volume-Phase Holographic Gratings (Review)," in ASP Conf. Ser. 195: Imaging the Universe in Three Dimensions, pp. 552-+, 2000.

2. H. Kogelnik, "Coupled wave theory for thick hologram gratings," Bell System Tech. J. 48(9), p. 2909, 1969.

3. D. Guzman, S. Blais-Ouellette, M. Bonati, and A. Elgamil, "A versatile wavelength-dependent spectrophotometer for efficiency measurements of ccd and cryogenic gratings." These proceedings. 